Notre Dame Journal of Formal Logic

Volume 23, Number 2, April 1982

\title{
Game-Theoretical Semantics: Insights and Prospects
}

\author{
JAAKKO HINTIKKA
}

1 Introductory The paradigm problem for game-theoretical semantics $(G T S)$ is the treatment of quantifiers, primarily logicians' existential and universal quantifiers. As far as the uses of quantifiers in logic and mathematics are concerned, the basic ideas codified in GTS have long been part and parcel of logicians' and mathematicians' folklore. Everyone who has taken a serious course in calculus remembers the definition of what it means for a function $y=f(x)$ to be continuous at $x_{0}$ : it means that, given a number $\delta$ however small, we can find $\epsilon$ such that $\left|f(x)-f\left(x_{0}\right)\right|<\delta$ given any $x$ such that $\left|x-x_{0}\right|<\epsilon$ (cf. [23], p. 186). The most natural way of making this jargon explicit is to envisage each choice of the value of an existentially bound variable to be my own move in a game and each choice of the value of a universally bound variable a move in the same game by an imaginary opponent. The former is what is covered by such locutions as "we can find", whereas the latter is what is intended by references to what is "given" to us. This is indeed what is involved in the continuity example. For what the above " $\epsilon-\delta$ definition" of continuity says is precisely

(1) $(\delta)(\exists \epsilon)(x)\left[\left(\left|x-x_{0}\right|<\epsilon\right) \supset\left(\left|f(x)-f\left(x_{0}\right)\right|<\delta\right)\right]$.

Here "we can find" corresponds to the existential quantifier " $(\exists \epsilon)$ " and the locution "given" to the universal quantifiers " $(\delta)$ " and " $(x)$ ". Game-theoretical treatment of the two quantifiers is to all intents and purposes just a systematization of the ideas involved in this example.

Logicians have even introduced a name for the functions that embody my strategy in choosing values for existentially bound variables. These are what is meant by Skolem functions in logic. Using $s(z)$ as such a function, we can for instance express what (1) says by asserting that a suitable Skolem function exists for my choice of $\epsilon$, i.e., asserting that 
(2) $(\exists s)(\delta)(x)\left[\left(\left|x-x_{0}\right|<s(\delta)\right) \supset\left(\left|f(x)-f\left(x_{0}\right)\right|<\delta\right)\right]$.

GTS will also incorporate this role of Skolem functions.

As a matter of historical fact, however, logicians have typically resorted to game-theoretical conceptualizations only when there is no hope of dealing with the semantics (model theory) of a branch of logic by means of the usual Tarski-type truth-definitions. ${ }^{1}$ The most conspicuous cases in point are the theory of partly ordered quantifiers ${ }^{2}$ and the theory of so-called game quantifiers (see [77], [62], [3], esp. pp. 242-254, and [69]). It is my thesis that game-theoretically inspired conceptualizations have much to offer in other parts of logical studies as well. An especially neat case in point is offered by Gödel's functional interpretation of first-order arithmetic. ${ }^{3}$ As Dana Scott first pointed out, ${ }^{4}$ by far the most natural way of looking at it is in game-theoretical terms. Other examples are found later in this paper and in the relevant literature.

2 Simple formal languages The basic ideas of GTS can be introduced most naturally by reference to formal first-order (quantificational) languages. ${ }^{5}$ The game-theoretical treatment of such a finite formal first-order language $L$ can be explained in a nutshell as follows. We assume that $L$ is an interpreted language. (Otherwise we cannot meaningfully speak of the truth or falsity of its sentences.) Being interpreted means that we are given some domain of individuals $D$ on which all the nonlogical constants of $L$ are interpreted. This in turn means that each atomic sentence (including each identity) that can be formed from the nonlogical constants of $L$, plus the names of the members of $D$, has a definite truth value, true or false. This is comparable with those clauses in a Tarski-type truth definition which govern atomic sentences. The function of a game-theoretical truth definition is the same as that of the other (recursive) clauses of a Tarski-type truth-definition, viz., to extend the notions of truth and falsity to all nonatomic sentences.

This is done in GTS in a way essentially different from Tarski's methods, however. It is done by associating with each well-formed sentence $S$ of any language $L^{\prime}$ a two-person zero-sum game $\mathrm{G}(S)$, where $L^{\prime}$ is $L$ extended by adjoining a finite number of names of members of $D$. We shall call the two players myself and nature. The definition of $\mathrm{G}(S)$ is as follows:

(G.A) If $A$ is atomic, then I have won $\mathrm{G}(A)$ and nature has lost if $A$ is true. If $A$ is false, nature has won and I have lost.

(G.\&) $\mathrm{G}\left(S_{1} \& S_{2}\right)$ begins by nature's choice of $S_{1}$ or $S_{2}$. The rest of the game is $\mathrm{G}\left(S_{1}\right)$ or $\mathrm{G}\left(S_{2}\right)$, respectively.

(G.v) $\quad \mathrm{G}\left(S_{1} \vee S_{2}\right)$ begins by my choice of $S_{1}$ or $S_{2}$. The rest of the game is $\mathrm{G}\left(S_{1}\right)$ or $\mathrm{G}\left(S_{2}\right)$, respectively.

(G.U) $\mathrm{G}((x) S(x))$ begins by nature's choice of a member of $D$. Let the name of the member chosen be " $b$ ". The rest of the game is then $\mathrm{G}(S(b))$.

(G.E) $\mathrm{G}((\exists x) S(x))$ is defined likewise except that $b$ is chosen by myself.

(G. ) $\quad \mathrm{G}(\sim S)$ is played like $\mathrm{G}(S)$ except that the roles of the two players (as defined by these rules) are interchanged. 
These rules are said to define semantical games. ${ }^{6}$

In (G.E) and (G.U) it is not assumed that the individual chosen already has a name. Rather, it is assumed that if it does not, the players will give it a proper name, thus extending the given language by adjoining to it one proper name of a member of $D$.

But what do these games have to do with semantics? They can be brought to bear on semantics by showing how they can be used to define the crucial concept of truth. GTS is truth-conditional, even though it works in a way different from (and even opposite to) Tarski-type theories of truth.

The truth of a sentence $S$ of $L$ can be defined as the existence of a winning strategy in $\mathrm{G}(S)$ for myself, i.e., a way of choosing my moves such that I end up winning no matter what nature does. The falsity of $S$ likewise means that nature has a winning strategy in $\mathrm{G}(S){ }^{7}$

Since the output of each application of the rules formulated above is simpler than its input (since it contains at least one logical symbol less than the input), our semantical games will always come to an end, after a finite number of moves, in the form of an atomic sentence. Hence (G. $A$ ) suffices to define winning and losing for all cases. For the same reason, the rules of semantical games can be considered rules of semantical analysis.

It is easily seen that in the simple languages we are currently considering, the game-theoretical definition of truth just presented coincides with the usual Tarski-type one. It can be seen almost equally easily that in many circumstances it offers interesting possibilities of modifying our usual truth definitions or otherwise putting them into a wider perspective. Suffice it here to mention only one such line of thought. Truth means in gametheoretical semantics that there is a winning strategy for myself, and falsity means the existence of a similar strategy for my opponent (nature). But who says that either one of us has a winning strategy? The law of excluded middle says so. On the basic game theory we now see that this law is by no means trivial or unproblematic. For in general it is not a foregone conclusion that there should exist a winning strategy for either one of the two players in a zero-sum two-person game. ${ }^{8}$ When one exists, the game is said to be determinate. From game theory we know that the determinateness of a game is usually a highly nontrivial result (or assumption). Indeed, determinateness assumptions for certain infinite games have recently played an important role as potential axioms in the higher reaches of axiomatic set theory. ${ }^{9}$ But even apart from such sophisticated situations, determinateness (and hence the law of excluded middle) can fairly easily fail.

Thus the principle of excluded middle is at once put into an interesting general perspective by $G T S$.

3 Extensions and generalizations in formal languages Several of the many interesting further developments of GTS can be discussed by reference to games connected with formal (but interpreted) first-order sentences or with sentences in various natural extensions of formal first-order languages.

Some of the further developments are:

(i) The existence of a winning strategy for either player in a given $\mathrm{G}(S)$ can be expressed in the form of an explicit higher-order sentence. This sentence 
asserts simply the existence of the relevant Skolem functions. What this means is that GTS effects a translation of first-order languages into higher-order languages. For instance, (1) translates into (2),

(3) $(x)(\exists y) M(x, y)$

translates into

(4) $(\exists f)(x) M(x, f(x))$,

and

(5) $(x)(\exists y)(z)(\exists u) M(x, y, z, u)$

translates into

(6) $(\exists f)(\exists g)(x)(z) M(x, f(x), z, g(x, z))$.

(I am assuming here that there are no quantifiers in $M$ and that propositional connectives are given the classical interpretation.)

The relation of (6) to (5) (and in general the relation of the higherorder translation of a first-order sentence which game-theoretical semantics gives us to its original) can be taken to exemplify the difference of a game in its normal form (game as a choice of a strategy) to the same game in its extensive form (for the distinction, see [68], pp. 76-84). Conversely, this illustration of familiar game-theoretical notions serves to bring their import into sharper perspective.

(ii) The interpretation of first-order languages in higher-order ones outlined in (i) above can be varied in different ways. These variations will result in many variants of nonclassical logic. One is to allow the game to be split into subgames, each of which is played out, whereupon one or both of the players reveal the strategy they used in the subgame. ${ }^{10}$ The functions which embody the strategies of the players in the overall game depend, hence, on the functions which incorporate players' strategies in the subgame. In other words, they are higher-order functions (functionals). Accordingly, the translations of first-order languages described in (i) above can now be higher-order sentences rather than just second-order ones. In brief, it is seen in this way that GTS is closely related to the various functional interpretations that can be given to first-order languages and theories (see note 3 above).

(iii) Such subgames are especially natural vehicles in the interpretation of conditionals. A subgame is first played with the antecedent $X$ of a conditional $(X \supset Y)$ (with roles exchanged), and only if nature manages to verify $X$ do the players move over to the game $\mathrm{G}(Y)$, where nature's winning strategy in the first subgame is known to myself. This is in excellent agreement with our ideas of the conditionality of conditionals; the consequent $Y$ enters the stage only if the antecedent has turned out to be true, and does so in a way which depends on the way $X$ has been verified.

There nevertheless remain several different ways in which this gametheoretical interpretation of conditionals can be carried out. (For one of them, see [36].)

(iv) In order to preserve classical logic, the values of our function 
variables (Skolem functions, such as the function variables in (2), (4), and (6)) have to include quite complex (noncomputable) functions (see [64], [65], and [52]). Since these functions are supposed to embody players' actual strategies, it nevertheless seems natural to restrict these functions to computable ones. This pushes our logic away from the classical one in another direction.

(v) The relation of the resulting interpretations to the classical one is essentially an instance of the relation of nonstandard to standard interpretations of higher-order logic in Henkin's sense. ${ }^{11}$ The only difference is that Henkin considers somewhat different closure conditions imposed on the values of higher-order variables than those that result from restricting the values of our function variables to computable ones. If we look away from this inessential qualification, we can say that GTS serves to extend the standard-nonstandard contrast from higher-order logics to the first-order one.

(vi) If the changes (ii)-(iv) are combined with a requirement of computability also for the moves for propositional connectives and a suitable rule for conditionals is chosen (cf. (iii) above), we obtain Gödel's famous functional interpretation of first-order languages (see note 3 above).

(vii) One of the first questions a game theorist is likely to ask is whether our semantical games are games with perfect information or not. Classical logic presupposes that semantical games are characterized by perfect information. This assumption is easily modified, however, especially in the case of quantifier rules. The result is then a kind of new logic which has been independently studied by a handful of logicians, viz., the theory of finite partly ordered (e.g., branching) quantifiers. ${ }^{12}$ There are several interesting facts about the theory of such quantifiers which make this theory an interesting test case in logical and semantical theory. First, it is (almost) as strong as the entire second-order logic (with standard interpretation), in the sense of having an equally difficult decision problem (see [28] and [54]). Second, the usual Tarski-type truth-definitions do not work in the theory of partly ordered quantifiers (see [4]).

The simplest branching quantifier prefixes reduce to linear prefixes. For instance,

$$
\begin{aligned}
& (\exists x) \\
& (y)
\end{aligned} M(x, y)
$$

reduces to

$(\exists x)(y) M(x, y)$.

However, more complex types of prefixes are not equivalent with any linear ones. For instance, the "Henkin quantifier" illustrated by

$$
\begin{aligned}
& (x)(\exists y)>M(x, y, z, u) \\
& (z)(\exists u)
\end{aligned}
$$

has no first-order linear equivalent (for a discussion of such reduction problems, see [79]). 
In all cases, we of course have a second-order translation. Thus (9) is equivalent with

$(\exists f)(\exists g)(x)(z) M(x, f(x), z, g(z))$.

This illustrates how informational dependencies among quantifiers are reflected by the arguments of the corresponding Skolem functions. (With (9)-(10) you may compare (5)-(6) above.)

(viii) Because of the failure of Tarski-type truth-definitions for partially ordered quantifier sentences (the second of the results mentioned in (viii) above), mathematical logicians have been forced to resort to game-theoretical conceptualizations in the study of partially ordered quantifiers. As was already mentioned, there is another direction in which they have sporadically done so, viz. in certain cases in which (in our terminology) a semantical game can go on to infinity (see [34] and [50]). Since our game rules are rules of semantical analysis, this means that one cannot define truth recursively from bottom up à la Tarski, for there is no bottom on which to build a recursive definition. This is no obstacle to game-theoretical truth-definitions, however, and on the contrary leaves open all sorts of interesting possibilities for setting up the definition of winning or losing (or neither) in the case of infinite plays of a semantical game. It remains to be seen how useful the general theory of infinitely deep logics is that can be based on this idea. In any case, it has the philosophical interest of being the first clear-cut general modern realization of Leibniz's idea of infinite analysis in logic (see [38], esp. p. 272).

(ix) There is no reason why the successive choices of individuals could not be made from different subsets of $D$. This means modifying the received notion of model (for first-order languages). The result is Rantala's notion of urn model. ${ }^{13}$ It has already had interesting applications in Rantala's theory of definability and in Hintikka's solution of the problem of "logical omniscience" in epistemic logic. ${ }^{14}$ We can now see that game-theoretical truthdefinition can be used in the theory of urn models without any changes (other than those that define urn models).

(x) Game-theoretical semantics is easily extended to intensional logics by using the well-known possible-worlds semantics for intensional logics as an interim step. The main novelty is that at each move the players are now considering, not only a sentence $S^{\prime}$, but also a world $w_{0}$ (a member of the frame with respect to which the original sentence is to be interpreted). The stronger of each pair of interrelated intensional operators (necessity, knowledge, belief, obligation, etc.) marks nature's move, the weaker (possibility, epistemic possibility, compatibility with one's belief's, permission, etc.) my move. A move consists in a selection of one of the worlds, say $w_{1}$, alternative to $w_{0}$. At the next move, the players will consider $w_{1}$ instead of $w_{0}$.

In brief, intensional operators are quantifiers ranging over alternative worlds. (This incidentally serves to highlight once again the fundamental importance of the alternativeness relation in intensional semantics.)

It turns out that we may then have to heed possible informational independencies between quantifier moves and moves connected with inten- 
sional operators (see [6]). What is more, informational dependencies need not be in such cases transitive; see [45]. (Any game theorist will tell you that the information sets involved in an arbitrary game are usually not transitive.) Hence not even branching structures suffice for the semantical representation of all the sentences which are naturally considered in this direction.

4 Transition to natural languages In spite of all these potentialities of game-theoretical semantics in formal languages, its most remarkable applications are to natural languages. The transition is based on the following idea: prima facie, there is no counterpart in natural languages to the procedure of substituting names of individuals chosen by the players for bound variables, for there are no variables in natural languages. The answer is that we can think of proper names for such individuals substituted for entire quantifier phrases. They include in the first place phrases of the following forms:

(11) some $X$ who $Y$

an $X$ who $Y$

every $X$ who $Y$

each $X$ who $Y$

any $X$ who $Y$.

Instead of "who", we can of course have in (11) some other wh-word.

This idea, in combination with the heuristic idea that a semantical game is an attempt on my part to verify a sentence against the schemes of recalcitrant nature, enables us to anticipate the game rules for the main English quantifier phrases. They are illustrated by the following special cases of the rules:

(G.some) (special case) If the game has reached the sentence

$$
X \text { - some } Y \text { who } Z-W \text {, }
$$

then an individual may be chosen by myself from that subdomain which consists of persons. If this individual does not have a proper name, it is given one. Let the proper name of the individual chosen be " $b$ ". The game is then continued with respect to

$$
X-b-W, b \text { is a } Y \text {, and } b Z .
$$

For simplicity, it is assumed here and in the next four rules that "who" occupies the subject position in "who $Z$ ". It is assumed in the present special cases of (G.some) and (G.any), for the time being, that the main verb in "who $X$ " is in the singular. ${ }^{15}$

From $(* *)$ it is seen how the name of the individual chosen replaces the whole quantifier phrase in $(*)$.

(G.an) (special case) As in the corresponding case of (G.some) except that "a(n)" replaces "some".

(G.every) (special case) As in the corresponding case of (G.some) except that $b$ is chosen by nature, that "every" replaces "some" in (*), and that the game is continued with respect to 
(***) $\quad X-b-W$ if $b$ is a $Y$ and $b Z$.

(G.each) (special case) As in the corresponding case of (G.every), except that "each" replaces "every".

(G.any) (special case) As in the corresponding case of (G.every), except that "any" replaces "every".

In spite of their simplicity, these rules codify nontrivial theses. For instance, (G.any) incorporates the claim that "any" is univocal in English and that it always has basically the force of a universal quantifier. This contradicts most of the recent views of linguists on "any", and puts an onus on me to provide an account of the many examples in which "any" prima facie has the force of an existential quantifier. ${ }^{16}$

Subject to explanations to be given later, the general setup and the rules for propositional connectives are similar to what we found in formal languages. We are still dealing with two-person zero-sum games, and a game $\mathrm{G}(S)$ can still be thought of heuristically as an attempt on the part of one of the players ("myself") to verify the sentence $S$ with which the game starts against the opposition of nature. At each stage of the game, some one sentence $S^{\prime}$ is being considered on which the players' next move depends. As before, some primitive vocabulary is assumed to be given, interpreted on a given domain. It is not as unproblematic as in the case of formal languages that the game comes to an end in a finite number of moves. This can be shown to be possible to guarantee, however, by small additions to our game rules, at least for the fragments of English we are interested in here.

As compared with formal languages, one striking novelty here is that the individual chosen by one of the players at each move is chosen from a subdomain of the total supply of individuals we can quantify over. A logician would say that we are dealing with many-sorted quantification. This fact will make more difference than perhaps first meets the eye (see below, Section 10).

Winning and losing can be defined as of old, and so can truth and falsity be defined as the existence of winning strategies for myself and for nature, respectively.

Negation can be dealt with in terms of role-swapping precisely as in the case of formal languages. ${ }^{17}$ The rules for conjunction and disjunction can be carried over without major changes for the uses of "and" and "or" to connect clauses, and they can be extended in an obvious way to those uses in which they connect phrases.

5 Amplifications, developments, and extensions These basic ideas of GTS as applied to natural languages can be developed further and extended in several different directions. In some cases, discrepancies between semantical games played with formal languages and those played with natural languages differ in important ways which can be recognized and which necessitate interesting further conceptualizations; in others, merely spelling out what is intended in our game rules prompts fascinating insights; in certain further cases, the extensions of GTS as applied to formal languages have counterparts in the realm of natural-language semantics; and in still others, extensions of 
game-theoretical treatment to other parts of natural languages lead to interesting problems. Here I can only discuss a selection of this embarrassment of riches.

\section{Differences between formal and natural languages and their consequences}

Among the important differences between formal and natural languages, viewed from the vantage point of GTS, there are the following:

(i) Rule-ordering: In formal languages, the sentence reached in the course of the game always determines what happens next. (The only exceptions envisaged above were partly ordered quantifier structures; but they are of course per definitionem the cases in which the order does not matter.) In contrast, in natural languages, several different rules may apply to the same sentence. Moreover, the order of application of the different rules has so far been left completely open. For instance, consider $(*)$ in the special case of (G.some) formulated above. There may be structures in $X, Y, Z$, or $W$ which would justify applying another game rule to $(*)$, or justify applying (G.some) to $(*)$ in some other way.

Is this freedom a vice or a virtue? If it is vice, the same vice is instantiated with vengeance by such theories as Montague grammars and Chomsky's Aspects theory. ${ }^{18}$ Both allow for even more ambiguities than result from the open ordering of our game rules. Hence it is at the very least less than obvious that the underdetermination of the order of different game rules is a blemish in GTS.

However, the correct course is fairly clearly to acknowledge that too much freedom has so far been left for rule ordering in my semantical games, and to try to formulate ordering principles which are calculated to govern this order. They constitute a new ingredient in GTS in natural languages, and they don't have any counterpart in the game-theoretical treatment of formal languages. They have already turned out to be an extremely powerful tool in explaining a large variety of linguistic phenomena.

There are two kinds of ordering principles, general and specific ones. The most important principles among the former are (O.comm) and (O.LR) which say, respectively, that a rule must not be applied to a lower clause if it can be applied to a higher one, and that in one and the same clause the rules are applied left to right. ${ }^{19}$

These general principles can be overruled by specific ones. They are exemplified by (O.any), which says that (G.any) has priority over the game rules for negation, conjunction, conditionals, as well as over modal rules (in the narrow sense which excludes rules for epistemic notions and for other propositional attitudes), and by (O.each) which says that (G.each) has priority over the other quantifier rules as well as over the rules for propositional connectives.

These ordering principles, and others like them, serve to explain a wide variety of semantical phenomena. A modest example is Quine's pair of sentences:

(12) If everybody comes to the party, I'll be surprised.

(13) If anybody comes to the party, I'll be surprised. 
The meanings of (12) and (13), and hence the difference between the two, are explainable on the basis of the fact that (G.any) has priority over (G.if) but (G.every) does not. ${ }^{20}$

Here we are already beginning to see how the sentences are to be analyzed in GTS in which "any" appears to have the force of an existential quantifier.

(ii) Scope vs. ordering: A related difference between formal and natural languages is that in formal languages the scope of each quantifier is indicated explicitly. In contrast, the concept of scope does not even make sense in a natural language. For in such a language a quantifier phrase can in principle have pronouns or anaphoric the-phrases referring back to it arbitrarily far later in the same sentence and even in the same discourse. Instead of the notion of scope we have to deal with the notion of rule ordering, which serves many of the same purposes as scope does in formal languages. This changes in several respects the situation in the semantics of natural languages as compared with the semantics of formal ones.

(iii) In formal languages, there are (as Frege and Russell emphasized) at least four different counterparts to the one and only verb for being in many natural languages ("is" in English, "ist" in German, etc.) (see [37] and the references therein). They include the following:

(a) the "is" of identity, exemplified by "Sue's brother is Jack" and formalized by "=";

(b) the "is" of predication (the copula), exemplified by "Sue is blond" and formalized by predicative juxtaposition;

(c) the "is" of existence, exemplified by "God is" and "there are Basques in California" and formalized by the existential quantifier;

(d) the "is" of general implication, exemplified by "A whale is a mammal" and formalized, e.g., by " $(x)$ ( $x$ is a whale $\supset x$ is a mammal)."

Does this distinction imply that natural-language words like "is" and "ist" are ambiguous, as Frege and Russell maintained? In GTS, most rules don't take into account different kinds of uses of "is". Does this mean that GTS treats "is" as unambiguous? But if so, how can it do so and yet do justice to the insights of Frege and Russell? These questions are discussed in Section 9.

(iv) As was already pointed out, in natural languages we are dealing with many-sorted quantification theory. In formal languages, different sorts of quantifiers are distinguished from each other notationally. In contrast, in natural languages these sorts (ranges of different quantifiers) have to be gathered from sundry syntactical as well as semantical clues (see [47]). What these clues are is to be examined separately.

All of these four new developments (i)-(iv) have remarkable consequences for the general methodology of linguistics. They are studied in the next four sections of this paper.

7 Any-thesis and the limitations of generative grammars It turns out that (O.any) enables us to formulate a semantical criterion for the admissibility of "any" in a given context $X$-any- $Y$ in a fragment of English. ${ }^{21}$ This criterion, 
called the any-thesis, says (in a rough unqualified form) that "any" is acceptable in this context if and only if $X$-every- $Y$ is grammatical and different in meaning from $X$-any- $Y$. This criterion works well in a certain fragment of English. For instance, it explains at once why "any" can occur ceteris paribus in the antecedents of a conditional (as in (13)) but not in the consequent, as illustrated by the following pair of sentences:

(14) If Chris trained hard, she has beaten everybody by this time.

(15) *If Chris trained hard, she has beaten anybody by this time.

Competing explanations of the admissibility and nonadmissibility of "any" in different contexts mostly turn on some straightforward syntactical characteristic of those contexts, e.g., that they are governed by "if" or "not". This will not work, however. "Any" can be inadmissible in a context governed by "if", viz., when it occurs in the consequent:

(16) *If Chris trained hard, she has by this time won any match.

"Any" can be inadmissible when governed (logically speaking) by negation:

(17) *Not any girl has been dated by Bill.

But "any" can occur even in the consequent if there are other features of the context to create a semantical asymmetry between "any" and "every":

(18) If Chris trained hard, she can win any match.

Thus no simple competing explanation does justice to the obvious data on which the any-thesis is based.

The salient questions how far the any-thesis can be extended beyond the original fragment and what qualifications it needs are partly open. This openness does not impair its remarkable consequences, however.

First, it presents to us an important instance in which the (apparently syntactical) well-formedness (acceptability) of a sentence (string) turns on semantical considerations. This wreaks havoc with Chomsky's early program of explaining well-formedness in exclusively generative (and hence syntactical) terms.

Chomsky is now willing to allow other, subsidiary factors to enter into explanations of well-formedness. ${ }^{22}$ However, he still thinks that the generative component is the most important one for the purposes of an overall linguistic theory.

This belief is threatened by the fact, illustrated by the any-thesis, that our semantical rules apply much more widely than merely to well-formed (acceptable) strings of English words. Indeed, in order to reject $X$-any- $Y$ in those cases where $X$-every- $Y$ is acceptable, we have to compare the meanings of the two sentences and hence to assign a meaning to the ill-formed string $X$-any- $Y$. This illustrates a recurring phenomenon uncovered by GTS, viz., that syntactical well-formedness and semantical interpretability frequently don't go together. This deprives generative syntax of much of its central theoretical interest.

This line of thought can be pushed further. Given certain further 
assumptions, it can be proved that the set of grammatical sentences of English is not even recursively enumerable and hence not generable in any generative grammar. (See [30], esp. pp. 76-90, and [39].) This shows strikingly the limitations of generative grammar as a universal explanation of grammaticality. Chomsky has tried to shrug off such results as turning merely on various "imperfections" of natural languages. A case can nevertheless be made to the effect that, in reality, it is transformational grammar that is dealing with various fringe irregularities while game-theoretical semantics deals with mainline regularities. In this direction, GTS thus suggests extremely interesting general conclusions.

8 The failure of compositionality It is not the only direction, however, where interesting theoretical insights are forthcoming. The absence of scope indicators from natural languages implies among other things that naturallanguage discourse and natural-language sentences cannot always be analysed into component parts whose meanings can be considered in isolation. (If such a component expression $e$ could be identified and if it contained an existential quantifier, the scope of this quantifier would have to be restricted to $e$. But we have seen that the scopes of natural-language quantifiers cannot be restricted in principle.)

Hence we see that there is no hope of maintaining the so-called Frege Principle (the Principle of Compositionality) in the semantics of natural languages. ${ }^{23}$ This principle says that the meaning of a complex expression is a function of the meanings of its component parts. We have just seen, however, that in natural languages there are in some cases no candidates for the role of such component expressions.

In other ways, too, it can be argued that compositionality is a lost cause in natural-language semantics. It presupposes the kind of semantical contextindependence which would enable us to carry our semantical analysis of a sentence from inside out. We cannot hope to find such context-independence obtaining in general. Hence compositionality is bound to fail sometimes.

Since the game rules of GTS operate from outside in, they allow us to take into account even such context-dependencies as violate compositionality. Cases in point are applications in which (O.any) overrules (O.comm). There are other similar phenomena, however. They generate examples where the meaning of an expression is different in a given context from what it is independently of it. Here are some cases in point:

(19) Anybody can beat Jimmy.

(20) I am surprised if anyone can beat Jimmy.

(21) I doubt that anyone can beat Jimmy.

(22) Bill owns a donkey.

(23) If Bill owns a donkey, he beats it.

Among the approaches which rely on compositionality, there are those that use essentially Tarski-type truth-definitions and/or Tarski's so-called $T$-schema. What GTS strongly suggests is that such approaches are unsatisfactory. This is illustrated by the failure of Tarski-type truth-definitions for branching quantifiers (cf. Section 3, (vii)-(viii) above) and by the failure of one half of the $T$-schema: 
(24) "Anybody can become millionaire" is true if anybody can become a millionaire.

This is not even true (see [29]).

The transition from (9) to (10) illustrates the fact that (at least in some cases) we can restore compositionality by evoking higher-order entities (functions which embody my strategies in semantical games). It seems to me that this is the tacit strategy employed in Montague semantics. Montague is strongly committed to compositionality. However, the only way in which he can hope to abide by it is to make liberal use of higher-order conceptualizations.

There is a price to be paid here, however. The higher-order entities we have to evoke in this "type-theoretical ascent" are much less realistic philosophically and psycholinguistically than our original individuals. Hence the ascent is bound to detract from the psycholinguistic and methodological realism of one's theory.

9 The failure of the Frege-Russell ambiguity claim $^{24}$ Consider what the game played on the following sentence will look like:

(25) Jack is a boy who plays chess.

The game rule applicable here is (G.an), with $X=$ Jack is, $Y=$ boy, $Z=$ plays chess, and $W=$ empty. The output sentence is then of the form

(26) Jack is John Jr., John Jr. is a boy, and John Jr. plays chess.

Nothing could be simpler. But the step from (25) to (26) is strange when considered from the vantage point of the Frege-Russell claim that "is" is multiply ambiguous. For in (25) the first "is" is clearly an "is" of predication, whereas in (26) the selfsame first "is" (part of the very same $X=J a c k$ is) has to be classified as an "is" of identity. What this means is that the FregeRussell ambiguity claim does not apply to some occurrences of "is", that "is" cannot be said to be ambiguous between the "is" of identity and the "is" of predication, if GTS is the right semantics of English.

Other examples show that the other parts of the Frege-Russell ambiguity claim cannot be upheld, either, in GTS. For instance, one way of dealing with sentences like

(27) A whale is a mammal

is to construe it as an ellipsis for

(28) If anything is a whale, it is a mammal.

Here the second "is" will be identical with the "is" of (27). In treating (28) in GTS, it must be taken to be on a par semantically with any other typical occurrence of the "is" of predication. Hence we cannot maintain an ambiguity between the "is" of predication and the "is" of class-inclusion, either.

This observation has important consequences. The Frege-Russell ambiguity claim is codified in the usual formalism of formal first-order languages. In this formalism, the four allegedly different senses of "is" are expressed in 
entirely different ways. Hence anyone who uses first-order languages (quantification theory) as his or her framework of semantical representation is committed to the Frege-Russell ambiguity claim. This makes bedfellows of as different philosophers, logicians, and linguists as the early Wittgenstein, Quine, Davidson, George Lakoff, and Chomsky. Hence the failure of the Frege-Russell claim in GTS has repercussions for several different approaches to logical and linguistic semantics. Even if you don't yet at this point believe in the superiority of GTS over the use of conventional first-order languages as a canonical notation in semantics, it certainly offers a consistent alternative to the more traditional kinds of semantics. We have, then, a most remarkable situation on our hands in that some of the most central concepts of all semantics-ambiguity, number of readings, etc.-turn out to be relative to one's preferred framework of semantical representation. This has interesting consequences for our ideas of the methodology of semantics in general.

Of course, there are on any view differences between different uses of "is". In GTS, too, distinctions have to be made between different kinds of primitive sentences containing "is". Notice, however, that these differences are not due to different meanings of this word, but to differences in the contexts in which it occurs. Moreover, in certain nonatomic contexts the distinction cannot even be made (as we saw).

One interest which the failure of the Frege-Russell ambiguity claim has is due to the fact that virtually no major philosophers before Frege (except to some extent $\mathrm{Mill}^{25}$ and De Morgan ${ }^{26}$ ) relied on the ambiguity. Hence GTS should make the ears of historians of philosophy perk up: it promises a framework for discussing the logic of being which is less anachronistic than the conventional Frege-Russell logic. ${ }^{27}$

10 GTS and the doctrine of categories This historical dimension can be pursued further (with this section, cf. [47]). It was noted earlier that in GTS we are dealing with many-sorted theory rather than a traditional onesorted one. But how is the "sort" (subdomain) determined from which the two players are making their choices? In order to answer this question, consider a generalization of our special case of (G.some) (Section 4, above). What are the clues that the players can use here to decide which subdomain the choice of the individual $b$ is made from? The most obvious one is the wh-word which occurs in the quantifier phrase. (Among them, I am here including "that" which is of course merely the relative-clause counterpart to "what".) If it is "who", the choice is between persons; if "where", between locations in space; if "when", between moments (or periods) of time; and so on. Some wh-words, especially "that" (or "what"), cover more than one subdomain, and some domains are reached only by means of a prepositional phrase (which contains a wh-word). Apart from these irregularities, however, there is a rough equivalence between the ranges of English quantifiers and wh-words (plus certain wh-phrases) in English.

However, the whole relative clause can be missing. Then the meaning of $Y$ (in (*), see (G.some)) will have to tell the players which subdomain the choice is made from. The need of this clue is seen also from the fact that if $Y$ is empty (missing), the wh-word itself has to be amplified so as to convey 
the necessary information, becoming, e.g., "someone", "somewhere", "sometime", etc. As a special case, we therefore obtain a semantical classification of the primitive predicates of English which goes together with our earlier distinctions.

Hence we apparently obtain a one-to-one correlation between several different distinctions. They are distinctions between

(29) (i) different wh-words (and phrases);

(ii) certain semantically determined classes of primitive predicates;

(iii) the (widest) domains of quantification.

Since each application of the quantifier rules introduces an occurrence of "is", we also have a correlated distinction between

(iv) certain different uses of "is".

The correlation of these four distinctions has a familiar ring to a historian of philosophy. The view we have arrived at is mutatis mutandis identical with Aristotle's doctrine of categories. Aristotle's several explanations of his doctrine correspond neatly to (i)-(iv). ${ }^{28}$ We can now also solve the two main perennial problems that arise in interpreting Aristotle's doctrine: Which distinction (i)-(iv) did Aristotle "really" mean? If he meant more than one, why should they go together? Now we can see that he could have meant all of them together, and that their going together is a most natural part of the semantics of any natural language like English or the ancient Greek.

It turns out, however, that the theory summed up in the parallelism of the distinctions (i)-(iv) is only an approximation. The precise ways in which it breaks down, nevertheless, seem to have even greater systematic and historical interest than the approximation.

11 Other extensions and applications These dramatic developments do not exhaust the uses of GTS in logical and linguistic semantics and philosophical analysis. Among the further possibilities which will only be mentioned but not described here, there are the following:

(a) Several of the extensions mentioned in connection with formal languages have counterparts for natural languages, especially (ii)-(iii), (iv), (v), (ix), and (x).

(b) Among these, the extension of GTS to intensional and temporal languages seems to be especially promising. I have argued that a game-theoretical treatment of epistemic words is needed for a semantical theory of English wh-questions, especially multiple wh-questions (see [33] and [44]). Furthermore, I have suggested that GTS enables us to deal more effectively with several puzzling phenomena concerning tenses and other temporal discourse in English than any competing approach (see [48]).

(c) In a different direction, the subgame idea enables us to understand the mechanism of certain types of anaphora, particularly pronouns whose heads are quantifier phrases (see [36] and the references found there). This is illustrated by the following examples due essentially to Geach and Karttunen, respectively: 
(30) If Bill owns a donkey, he beats it.

(31) If you give every child a present for Christmas, some child will open it today.

(d) Another species of pronouns whose semantical behavior can be handled by means of GTS are the so-called "pronouns of laziness" (see [35]). They are the pronouns in which there is no coreferentiality with their heads, and hence no hope of handling then in terms of variables of quantification. They are instantiated by such Karttunen-type examples as

(32) Any man who gives his paycheck to his wife is wiser than any man who gives it to his mistress.

They can be analyzed in GTS, and the resulting explanation of the phenomenon of "laziness" can be extended to other cases, such as the Geach example

(33) John is not the only man who loves his wife.

(e) Likewise, GTS opens a new way of dealing with the anaphoric uses of the-phrases (definite descriptions). The main idea is to take them to represent choices from the finite set of individuals given in the beginning of a semantical game or selected by the players up to the time that the the-phrase in question is dealt with. This seems to yield for the first time a viable theory of such the-phrases. It also suggests a way of looking in a similar way at anaphoric pronouns.

(f) The ideas (b)-(d) can all be extended to discourse (text) semantics by means of the concept of subgame. In general, we can by means of GTS obtain a unified treatment of important parts of sentence semantics and discourse semantics. ${ }^{29}$

(g) Several aspects of the behavior of negation in English can be understood better by means of GTS.

(h) A large field of potential applications is constituted by the so-called nonstandard quantifiers, such as "many", "few", "several", "almost all", etc. Their behavior offers some of the best examples of some of the most interesting natural-language phenomena to which GTS has first focused our attention, for instance, informational independence. Together with the plural uses of such quantifier words as "some" and "any", they show convincingly how much more there is to the semantics of natural languages like English than the semantics of first-order logic.

These are only samples of what GTS can do. They also partly represent work which is still in progress rather than completed results. They are enough, however, to illustrate the great potentialities of this approach.

The main ideas of game-theoretical semantics can also be connected with the views of more than one major philosopher. Perhaps the most obvious connection is the affinity between semantical games and Wittgenstein's "language-games". ${ }^{30}$ There are also deep connections between my approach and Kant's views of logic and mathematics, especially the mathematical method (see [46], [49], and [27]). Neither relationship is discussed here, however. 


\section{NOTES}

1. There is a major exception to this neglect of game-theoretical conceptualizations by logicians. The complex of techniques which has in the last twenty years played a major role in systematic logical theory, especially in model theory, and which is called "backand-forth methods", is game-theoretical in spirit, and is occasionally given an explicitly game-theoretical formulation (see, e.g., [13] and [12], on "Ehrenfeucht Games"). This technique is largely equivalent with the distributive normal form technique of Hintikka;cf. [26] and [16]. Some of these connections are made explicit in [73].

2. For the first introduction of the idea, see Henkin [25]; for a selection of recent papers see [14], [79], [2], [53], and [54].

3. See Gödel [21] (and the English translation [22], with a bibliography of subsequent work along the same lines). Cf. [75], [19], [20], and [78].

4. Dana Scott, unpublished note on Gödel's functional interpretation, Stanford, 1968.

5. Most of the early work on GTS has been collected in [74]. The second essay reprinted there, J. Hintikka, "Quantifiers in logic and quantifiers in natural languages", comes perhaps closest to an introductory discussion.

6. As has been explained, semantical games are played on the domain of individuals which our interpreted language can be used to convey information about. (For instance, moves connected with quantifiers are choices of members of the domain $D$.) Semantical games are, hence, essentially different from so-called dialogical games, whose moves are utterances or other kinds of propoundings of sentences. This distinguishes the semantical games of GTS from, e.g., the dialogical games of Lorenzen (for them, see [56], and the literature referred to there, plus [76]). Even Lorenzen's and Lorenz's partial shift from "formal" to "material" games does not change the picture essentially. I have commented on the relation between the different kinds of games in [46]. As is pointed out there, semantical games and suitable dialogical games may in the last analysis perhaps be brought under the same roof after all.

7. Even though the game-theoretical concepts used here are almost self-explanatory (and hopefully familiar to the reader), a couple of brief explanations may be in order. A strategy of a player is a rule that tells the player in question what to do in each conceivable situation that may come up in the game. In this paper, only pure (nonprobabilistic) strategies are considered. By means of the concept of strategy, the whole game can always be reduced to a choice of a strategy by each player. This is known as the normal form of a game. If a strategy of a player in a two-person zero-sum game wins against any strategy of one's opponent, it is said to be a winning one.

8. Cf. here the classical paper by Gale and Stewart [18]; and cf. also [10].

9. This line of thought was opened by Mycielski in [67] and [66]. For samples and surveys of the latter work, see, e.g., [15] (with references); [57]; [58], especially pp. 807-814 (with further references); and [63], especially chapter 6.

10. Cf. in (ii)-(iii), [36], reprinted in [74], pp. 179-214.

11. See Henkin [24] (but cf. [1]); Hintikka [40] and [43] and the further literature referred to there.

12. See note 2 above. For applications to semantics, including natural language semantics, see [28] (reprinted in [74]; and Hintikka, "Quantifiers in natural languages: some logical problems" in [74], pp. 81-117, especially pp. 88-99; and [4]. 
13. Rantala [71], reprinted in [74], pp. 347-366.

14. See, respectively, [72] and [31], reprinted (and expanded) in [74], pp. 367-379.

15. Various further explanations are needed here. For instance, more has to be said of how anaphoric relations are determined in the output sentence of (G.some). Even more obviously, something ought to be said of what happens when the restrictions just mentioned are removed. A detailed discussion of these matters will not be attempted here, however.

16. For competing recent views on "any", cf., e.g., [51] and [55] (with further references to the literature).

17. Both in formal and natural languages negation can nevertheless be treated in a more informative way by giving explicit rules as to how the (semantical) negation (contradictory) of a given sentence can be formed syntactically. Such a treatment would be more informative, but it has not yet been attempted in print.

18. See [61] and [7], e.g., pp. 224-225 (note 9).

19. These ordering conventions clearly are closely related to various interesting linguistic principles, such as the cyclic principle of transformational grammarians and George Lakoff's "derivational constraints" in his generative semantics. Unlike the claims made for these principles, however, my ordering conventions admit exceptions.

20. This means that the "logical form" of (12) is $(x) F(x) \supset G$ while that of (13) is $(x)(F(x) \supset G)$, which can be rewritten as $(\exists x) F(x) \supset G$.

21. Cf. here, Hintikka, "Quantifiers in natural languages: some logical problems," pp. 81117 in [74], especially pp. 99-114; [39] (with further references); and [30]. Earlier, rules essentially tantamount to the any-thesis were formulated by Poutsma [70], and by Laurence R. Horn. (The last two references were pointed out to me by Lauri Carlson.)

22. Cf. [8], especially pp. 122-128; and [39].

23. With Section $8, \mathrm{cf}$. [41] and the literature mentioned there.

24. With Section 9, cf. [37].

25. Mill [60], vol. 2, chapter 4, §1.

26. De Morgan [11], pp. 49-50.

27. For instance, Kant has often been presented as a precursor of the Frege-Russell distinction. This is mistaken, as is argued in Hintikka [42]. Again, the Frege-Russell distinction was used as an essential ingredient of one's interpretive framework by a whole generation of students of ancient Greek philosophy (Cornford, Guthrie, Raven, Vlastos, Cherniss, Ryle, etc.). Only recently-and slowly-have scholars begun to point out how completely absent the distinction is from the thought of Plato, Aristotle, and their ilk, and how anachronistic the use of this distinction is; cf. [17], [59], and [9]. I suspect that many more scholars would have protested against the imposition of the Frege-Russell distinction on Plato and Aristotle if they had realized that the denial of the distinction does not mean a logical howler.

28. He uses different question words (and phrases) of the ancient Greek as names for his categories; he introduces categories as semantically determined classes of simple predicates; he treats categories as the widest genera of entities we can meaningfully consider together; and he frequently says that the several categories go together with different senses or uses of to einai, the Greek verb for being. 
29. For one highly interesting line of thought in this direction, see [5].

30. See [32], reprinted, pp. 1-26 in [74]; and cf. "Language-games for quantifiers," pp. 5382 in [27].

\section{REFERENCES}

[1] Andrews, P., "General models and extensionality," The Journal of Symbolic Logic, vol. 37 (1972), pp. 395-397.

[2] Barwise, J., "Some applications of Henkin quantifiers," Israel Journal of Mathematics, vol. 25 (1976), pp. 47-63.

[3] Barwise, J., Admissible Sets and Structures, Springer Verlag, Berlin and New York, 1975.

[4] Barwise, J., "On branching quantifiers in English," Journal of Philosophical Logic, vol. 8 (1979), pp. 47-80.

[5] Carlson, L., "Focus and dialogue games," in Cognitive Constraints on Communication, eds., L. Vaina and J. Hintikka, D. Reidel Publishing Company, Dordrecht, Holland, forthcoming.

[6] Carlson, L. and A. ter Meulen, "Informational independence in intensional context," pp. 61.72 in Essays in Honour of Jaakko Hintikka, eds., E. Saarinen et al., D. Reidel Publishing Company, Dordrecht, Holland, 1979.

[7] Chomsky, N., Aspects of the Theory of Syntax, The M.I.T. Press, Cambridge, Massachusetts, 1965.

[8] Chomsky, N., Rules and Representations, Columbia University Press, New York, New York, 1980.

[9] Dancy, R., "Toward a grammar of being in Aristotle," forthcoming.

[10] David, M., "Infinite games of perfect information," pp. 85-101 in Advances in Game Theory, eds., M. Dresher, L. S. Shapley, and A. W. Tucker (Annals of Mathematics Studies, Vol. 52), Princeton University Press, Princeton, New Jersey, 1964.

[11] De Morgan, A., Formal Logic, Taylor and Walton Publishers, London, 1847 (reprinted by The Open Court Company, London, 1926).

[12] Ebbinghaus, H. D., J. Flum, and W. Thomas, Einführung in die mathematische Logik, Wissenschaftliche Buchgesellschaft, Darmstadt, Deutschland, 1978.

[13] Ehrenfeucht, A., "An application of games to the completeness problem for formal theories," Fundamenta Mathematicae, vol. 49 (1960-61), pp. 129-141.

[14] Enderton, H. B., "Finite partially ordered quantifiers," Zeitschrift für mathematische Logik und Grundlagen der Mathematik, vol. 16 (1970), pp. 393-397.

[15] Fenstad, J. E., "The axiom of determinateness," pp. 41-61 in Proceedings of the Second Scandinavian Logic Symposium, ed., J. E. Fenstad, North-Holland Publishing Company, Amsterdam, Holland, 1971.

[16] Flum, J., "Distributive normal forms," pp. 71-76 in Proceedings of the Symposiums on Mathematical Logic in Oslo 1974 and in Helsinki 1975, eds., S. Miettinen and J. Väänänen, Helsinki, 1977. 
[17] Frede, M., Prädikation und Existenzaussage, Vandenhoeck und Ruprecht, Göttingen, 1967.

[18] Gale, D. and F. M. Stewart, "Infinite games with perfect information," pp. 245-266 in Contributions to the Theory of Games, vol. 2 (Annals of Mathematics Studies, Vol. 28), eds., H. W. Kuhn and A. W. Tucker, Princeton University Press, Princeton, New Jersey, 1953.

[19] Girard, J.-Y., "Une extension de l'interpretation de Gödel," pp. 63-92 in Proceedings of the Second Scandinavian Logic Symposium, ed., J. E. Fenstad, North-Holland Publishing Company, Amsterdam, Holland, 1971.

[20] Girard, J..Y., "Functional interpretation and Kripke models," pp. 33-57 in Logic, Foundations of Mathematics, and Compatability Theory (Part One of the Fifth International Congress of Logic, Methodology, and Philosophy of Science), eds., R. E. Butts and J. Hintikka, D. Reidel Publishing Company, Dordrecht, Holland, 1977.

[21] Gödel, K., "Über eine bisher noch nicht benützte Erweiterung der finiten Standpunktes," pp. 76-83 in Logica: Studia Paul Bernays dedicata, Editions du Griffon, Neuchâtel-Suisse, 1959.

[22] Gödel, K., "On a hitherto unexploited extension of the finitary standpoint," Journal of Philosophical Logic, vol. 9 (1980), pp. 133-142.

[23] Hardy, G. H., A Course of Pure Mathematics, 7th Ed., Cambridge University Press, Cambridge, England, 1938.

[24] Henkin, L., "Completeness in the theory of types," The Journal of Symbolic Logic, vol. 15 (1950), pp. 81-91.

[25] Henkin, L., "Some remarks on infinitely long formulas," pp. 167-183 in Infinitistic Methods, Pergamon Press, Oxford, England, 1961.

[26] Hintikka, J., "Distributive normal forms in first order logic," pp. $48-91$ in Formal Systems and Recursive Functions, eds., J. N. Crossley and M. A. E. Dummett, NorthHolland Publishing Company, Amsterdam, Holland, 1965.

[27] Hintikka, J., Logic, Language-Games, and Information, Clarendon Press, Oxford, England, 1973.

[28] Hintikka, J., “Quantifiers vs. quantification theory,” Linguistic Inquiry, vol. 5 (1974), pp. 153-177.

[29] Hintikka, J., "A counterexample to Tarski-type truth-definitions as applied to natural languages," Philosophia, vol. 5 (1975), pp. 204-212.

[30] Hintikka, J., "On the limitations of generative grammar," pp. 1-92 in Proceedings of the Scandinavian Seminar on Philosophy of Language, vol. 1 (Filosofiska studier utgivna av Filosofiska Föreningen och Filosofiska Institutionen vid Uppsala Universitet, vol. 26), Uppsala, Sweden, 1975.

[31] Hintikka, J., "Impossible possible worlds vindicated," Journal of Philosophical Logic, vol. 4 (1975), pp. 475-484.

[32] Hintikka, J., "Language-games," pp. 105-125 in Essays on Wittgenstein in Honour of G. H. von Wright (Acta Philosophica Fennica, Vol. 28, nos. 1-3), eds., J. Hintikka et al., North-Holland Publishing Company, Amsterdam, Holland, 1976.

[33] Hintikka, J., The Semantics of Questions and the Questions of Semantics (Acta Philosophica Fennica, Vol. 28, no. 4). 
[34] Hintikka, J. and V. Rantala, "A new approach to infinitary languages," Annals of Mathematical Logic, vol. 10 (1976), pp. 95-115.

[35] Hintikka, J. and L. Carlson, "Pronouns of laziness in game-theoretical semantics," Theoretical Linguistics, vol. 4 (1977), pp. 1-29.

[36] Hintikka, J. and L. Carlson, "Conditionals, generic quantifiers, and other applications of subgames," pp. 1-36 in Formal Semantics and Pragmatics of Natural Languages, eds., F. Guenther and S. J. Schmidt, D. Reidel Publishing Company, Dordrecht, Holland, 1979.

[37] Hintikka, J., “'Is', semantical games, and semantical relativity," Journal of Philosophical Logic, vol. 8 (1979), pp. 433-468.

[38] Hintikka, J., "Leibniz on plenitude, relations, and the 'Reign of Law'," pp. 259-286 in Reforging the Great Chain of Being, ed., S. Knuuttila, D. Reidel Publishing Company, Dordrecht, Holland, 1980.

[39] Hintikka, J., "On any-thesis and the methodology of linguistics," Linguistics and Philosophy, vol. 4 (1980), pp. 101-122.

[40] Hintikka, J., "Standard vs. nonstandard logic: higher order, modal, and first-order logics," pp. 283-296 in Modern Logic: A Survey, ed., E. Agazzi, D. Reidel Publishing Company, Dordrecht, Holland, 1980.

[41] Hintikka, J., "Theories of truth and learnable languages," pp. 37.57 in Philosophy and Grammar, eds., S. Kanger and S. Öhman, D. Reidel Publishing Company, Dordrecht, Holland, 1981.

[42] Hintikka, J., "Kant on existence, predication, and the ontological argument," Dialectica, vol. 35 (1981), pp. 127-146.

[43] Hintikka, J., "Is alethic modal logic possible?" Acta Philosophica Fennica, forthcoming.

[44] Hintikka, J., "New foundations of a theory of questions and answers," forthcoming.

[45] Hintikka, J., "On games, questions, and strange quantifiers," in Festschrift for Paul Grice, ed., R. Grandy, forthcoming.

[46] Hintikka, J., "Semantical games and transcendental arguments," in Theory of Argumentation, ed., E. Barth, Benjamins, Amsterdam, Holland, forthcoming.

[47] Hintikka, J., "Semantical games, the alleged ambiguity of 'is', and Aristotelian categories," forthcoming.

[48] Hintikka, J., “Temporal discourse and semantical games," Linguistics and Philosophy, forthcoming.

[49] Hintikka, J., "Transcendental arguments revived," in Philosophers on Their Own Work, eds., A. Mercier and M. Svilar, forthcoming.

[50] Karttunen, M., "Infinitary languages $N_{\infty}$ and generalized partial isomorphisms," pp. 153-168 in Essays on Mathematical and Philosophical Logic, eds., J. Hintikka et al., D. Reidel Publishing Company, Dordrecht, Holland, 1979.

[51] Klima, E. S., "Negation in English," pp. 246-323 in The Structure of Language, eds., J. A. Fodor and J. J. Katz, Prentice-Hall, Englewood Cliffs, New Jersey, 1964.

[52] Kreisel, G., "A note on arithmetic models for consistent formulae of the predicate calculus," pp. 39-49 in Proceedings of the XIth International Congress of Philosophy, vol. 14, Amsterdam and Louvain, 1953. 
[53] Krynicki, M., "On the expressive power of a language using the Henkin quantifier," pp. 259-265 in Essays on Mathematical and Philosophical Logic, eds., J. Hintikka et al., D. Reidel Publishing Company, Dordrecht, Holland, 1979.

[54] Krynicki, M. and A. H. Lachlan, "On the semantics of the Henkin quantifier," The Journal of Symbolic Logic, vol. 44 (1979), pp. 184-200.

[55] Ladusaw, W. A., Polarity Sensitivity as Inherent Scope Relations, Indiana University Linguistics Club, Bloomington, Indiana, 1980.

[56] Lorenzen, P. and K. Lorenz, Dialogische Logik, Wissenschaftliche Buchgesellschaft, Darmstadt, Deutschland, 1978.

[57] Martin, D. A., “Borel determinacy," Annals of Mathematics, vol. 102 (1975), pp. 363-371.

[58] Martin, D. A., "Descriptive set theory: projective sets," pp. 783-815 in Handbook of Mathematical Logic, ed., J. Barwise, North-Holland Publishing Company, Amsterdam, Holland, 1977.

[59] Mates, B., "Identity and predication in Plato," Phronesis, vol. 24 (1979), pp. 211-229.

[60] Mill, J. S., A System of Logic, 2 vol., ed., J. M. Robson, University of Toronto Press, Toronto, Canada, 1974.

[61] Montague, R., Formal Philosophy: Selected Papers of Richard Montague, ed., R. Thomason, Yale University Press, New Haven, Connecticut, 1974.

[62] Moschovakis, Y. N., "The game quantifier," Proceedings of the American Mathematical Society, vol. 31 (1971), pp. 245-250.

[63] Moschovakis, Y. N., Descriptive Set Theory, North-Holland Publishing Company, Amsterdam, Holland, 1980.

[64] Mostowski, A., "On a system of axioms which has no recursively enumerable arithmetic model," Fundamenta Mathematicae, vol. 40 (1953), pp. 56-61.

[65] Mostowski, A., "A formula with no recursively enumerable model," Fundamenta Mathematicae, vol. 42 (1955), pp. 125-140.

[66] Mycielski, J. and H. Steinhaus, "A mathematical axiom contradicting the axiom of choice," Bulletin de l'Academie Polonaise des Sciences, Series III, vol. 10 (1962), pp. 1-3.

[67] Mycielski, J., "On the axiom of determinateness," Fundamenta Mathematicae, vol. 53 (1964), pp. 204-224.

[68] Neuman, J. von and Morgenstern, Theory of Games and Economic Behavior, Princeton University Press, Princeton, New Jersey, 1953.

[69] Oikkonen, J., "Second order definability, game quantifiers, and related expressions," Societas Scientiarum Fennica Commentationes Physico-Mathematicae, vol. 48, no. 1 (1978), pp. 39-101.

[70] Poutsma, H., A Grammar of Late Modern English, I-II, Groningen, 1914.

[71] Rantala, V., "Urn models: A new kind of non-standard model for first-order logic," Journal of Philosophical Logic, vol. 4 (1975), pp. 455-474.

[72] Rantala, V., Aspects of Definability (Acta Philosophica Fennica, Vol. 29, nos. 2-3), North-Holland Publishing Company, Amsterdam, Holland, 1977. 
[73] Rantala, V., "Game-theoretical semantics and back-and-forth," pp. 119-151 in Essays on Mathematical and Philosophical Logic, eds., J. Hintikka et al., D. Reidel Publishing Company, Dordrecht, Holland, 1979.

[74] Saarinen, E., ed., Game-Theoretical Semantics, D. Reidel Publishing Company, Dordrecht, Holland, 1979.

[75] Spector, C., "Provably recursive functionals of analysis," pp. 1-27 in Recursive Function Theory: Proceedings of Symposia in Pure Mathematics, vol. 5, American Mathematical Society, Providence, Rhode Island, 1962.

[76] Stegmüller, W., "Remarks on the completeness of logical systems relative to the validity-concepts of P. Lorenzen and K. Lorenz," Notre Dame Journal of Formal Logic, vol. 5 (1964), pp. 81-112 (also in Stegmüller, W., Collected Papers, vol. 2, D. Reidel Publishing Company, Dordrecht, Holland, 1977).

[77] Svenonius, L., "On the denumerable models of theories with extra predicates," pp. 376-389 in The Theory of Models, eds., J. W. Addison, L. Henkin, and A. Tarski, North-Holland Publishing Company, Amsterdam, Holland, 1965.

[78] Troelstra, A. S., "Realizability and functional interpretations," pp. 175-274 in Metamathematical Investigations of Intuitionistic Arithmetic and Analysis (Lecture Notes in Mathematics, Vol. 344), ed., A. S. Troelstra, Springer Verlag, Berlin, Heidelberg, and New York, 1973.

[79] Walkoe, Jr., W. J., "Finite partially ordered quantification," The Journal of Symbolic Logic, vol. 35 (1970), pp. 535-555.

Department of Philosophy

Florida State University

Tallahassee, Florida 32306 